\title{
Epistemología y metodología de la investigación científica en la filosofía experimental de Galileo Galilei y Francis Bacon
}

\author{
Epistemology and methodology of the scientific investigation in the experimental \\ philosophy of Galilean Galilei and Francis Bacon
}

Ángel Gómez Navarro ${ }^{1}$

\begin{abstract}
RESUMEN
El presente estudio tiene como propósito desarrollar un estudio comparativo sobre los modos de acceder a la comprensión del mundo físico natural tal como se desprende de las teorías naturalistas y mecanicistas de Galileo Galilei y Francis Bacon. En efecto, la nueva racionalidad científica implicaba no solo la consideración de concepciones epistemológicas y cosmológicas, sino también metodológicas por las que la filosofía galileana y baconiana las presentan como condición de posibilidad para la obtención de aquellos conocimientos relevantes. En tal sentido, estudiar la constitución del nuevo método de indagación $y$ los elementos que la configuran se constituye en un aporte importante, tanto para el conocimiento humano en general como para el conocimiento científico en particular.
\end{abstract}

\section{Palabras clave}

Epistemología, ciencia, método, experiencia, nueva filosofía

\begin{abstract}
The purpose of this study is to develop a comparative study about the manners of accessing the comprehension of the natural physical world as it is clear from the naturalistic and mechanistic theories of Galileo Galilei and Francis Bacon. In fact, the new scientific rationality implied not only the consideration of epistemological and cosmological conceptions, but also the methodological ones by which the Galilean and Baconian philosophy presents them as a condition of possibility for obtaining Relevant knowledge.
\end{abstract}

In this sense, to study the constitution of the new method of investigation and the elements that form it is constituted in an important contribution, so much for the human knowledge in general as for the scientific knowledge in particular.

\section{Keywords}

Epistemology, science, method, experience, new philosophy

\section{INTRODUCCIÓN}

El presente estudio tiene como propósito desarrollar un análisis comparativo sobre la nueva filosofía experimental desarrollada por el italiano Galileo Galilei (1564-1642) y promovida por el inglés Francis Bacon (15611626). Esta nueva filosofía implicó no solo la confrontación de concepciones cosmológicas tradicionales, sino también epistemológicas y metodológicas, estrechamente vinculadas a la experiencia sensible y al razonamiento inductivo-deductivo, por el que el pensamiento científico-filosófico de Galilei

1 Doctor y Magíster en Ciencias de la Religión por la Pontificia Universidad Gregoriana de Roma. Egresado del Doctorado en Ciencias de la Educación de la Universidad Privada Marcelino Champagnat y del Doctorado en Ciencias Sociales de la Universidad Nacional Mayor de San Marcos. Actualmente se desempeña como Director de la Escuela de Posgrado de la Universidad Femenina del Sagrado Corazón, Miembro del Grupo de Investigación EPISTEME de la Universidad Nacional Mayor de San Marcos y Becario del Doctorado en Filosofía en la Pontificia Universidad Católica del Perú. 
y Bacon establecen notables semejanzas, especialmente, en cuanto a las maneras de aproximarse al mundo natural, comprender y explicar objetivamente aquellos saberes que llegaron a constituirse en el soporte de la naciente racionalidad moderna. En tal sentido, estudiar la configuración epistémica de la nueva filosofía experimental, así como el respectivo método que la sustenta se constituyen en un aporte más que relevante, no solo para la historia de la ciencia occidental sino también para la construcción y reconstrucción del conocimiento humano en general.

\section{I}

Se parte desarrollando brevemente las características fundamentales de la cosmología dominante en el pensamiento científicofilosófico del siglo XVII. Se trataba de la cosmología aristotélico-tolemaica que había empezado a ser revisada críticamente un siglo antes por el clérigo polaco Nicolás Copérnico (1473-1543). Esa se sustentaba en la física de Aristóteles, quien había planteado una concepción dualista del cosmos. En efecto, el Estagirita consideraba a nuestro mundo terrenal como un mundo sublunar, es decir, situado por debajo de la esfera de la Luna y constituido por cuatro elementos: Tierra, agua, aire y fuego. Él sostenía que tanto la tierra como el agua tendían a caer o descender, por ser pesados, y que el aire y el fuego tendían a subir o ascender por ser livianos. Asimismo, existía otro mundo que estaba por encima de la esfera lunar y que se caracterizaba por ser eterno, perfecto, inmutable y formado por un quinto elemento o esencia, el éter. Por consiguiente, la tierra estaba al centro del universo y la justificación se hallaba en la pesantez de los elementos terrenales.

Por su parte, el egipciano Claudio Ptolomeo (Tolomeo), en su obra "Almagesto", también había sostenido que la tierra era el centro del universo y que el sol, la luna y los planetas giraban en torno a ella movidas por el primer móvil, el cual movía a todos los demás.
Esta cosmología estaba constituida de una explicación geométrica que no incluía ningún sustento experimental contrastable respecto del movimiento de los cuerpos celestes.

Sin embargo, dicha explicación fue criticada por Nicolás Copérnico, por presentar fallos en los principios axiomáticos, tal como se puede apreciar en la dedicatoria de su libro "De Revolutionibus" (1543) al Papa Paulo III. En efecto, ahí afirmaba que:

"[Los astrónomos tolemaicos] tampoco han podido descubrir o deducir lo más importante, esto es, la forma del mundo y la simetría de sus partes. Al contrario, la experiencia realizada por ellos ha sido como la de alguien que tomara de diversos lugares manos, pies, una cabeza, y otros miembros, muy bien trazados, puede ser, pero no para la representación de una misma persona; como tales fragmentos no se corresponderían unos con otros, lo que se compondría con ellos sería un monstruo más que un hombre. Por consiguiente, en el proceso de demostración o método -como se le denomina- se encuentra que aquellos que han empleado excéntricas o bien han admitido algo esencial o bien han admitido algo extraño y completamente fuera de lugar. Esto no les hubiera ocurrido, de haber seguido principios seguros. Pues si las hipótesis asumidas por ellos no fueran falsas, todo lo que se sigue de sus hipótesis se confirmaría más allá de toda duda". ${ }^{2}$

Un siglo después, Galileo retoma la discusión copernicana y logra defender y sustentar dicha teoría construyendo para ello una nueva filosofía sustentada en un nuevo procedimiento científico. En efecto, el pisano ofrece una nueva manera de comprender y explicar el

2 Traducción del inglés, disponible en: http://webexhibits.org/calendars/year-text-Copernicus.html El nuevo sistema heliocéntrico de Copérnico no podía sustentarse en la física aristotélica, pues defendía el movimiento de la tierra sobre su propio eje. 
funcionamiento mecánico de la naturaleza en donde el instrumento de observación será la clave para dicho cometido.

En su obra, El Ensayador, Galileo manifiesta la exclusividad de su conocimiento logrado, precisamente, en virtud del telescopio adaptado por él mismo: "No puede usted remediar el hecho, señor Sarsi, de que me haya sido dado a mí solo descubrir los nuevos fenómenos del cielo, y a nadie más que a mí. Esta es la verdad que ni la malicia ni la envidia pueden anular". Asimismo, en el Sidereus Nuncius (El mensajero de los astros) vuelve a manifestar haber visto el cielo con el telescopio y presenta lo observado con un lenguaje matemático $y$, aunque esto no fue aceptado por un grupo de académicos italianos, sí lo fue por el astrónomo alemán Johannes Kepler quien, sin haber contrastado con el telescopio, asintió confiando totalmente en la autoridad de Galileo, razón por la que Kepler fue cuestionado hasta que él mismo pudo confirmar por experiencia directa los aportes galileanos cuando tuvo acceso al telescopio, tal como consta en el folleto kepleriano publicado con el título de "Relación de las observaciones de los cuatro satélites errantes de Júpiter" (1610).

En sus Diálogos [de las cuatro jornadas], donde discute el sistema tolemaico y copernicano, Galileo dedica la primera jornada a negar la distinción aristotélica entre el mundo celeste (inmutable) y nuestro mundo terrenal (mutable), sosteniendo que no existe tal distinción, y para ello se remite precisamente al sustento empírico obtenido por el telescopio, potenciador de los sentidos. Esto cobra mayor sentido al considerar lo que el mismo Aristóteles había determinado con su afirmación: "No hay nada en el intelecto que antes no haya pasado por los sentidos". Es decir, los datos de los sentidos son la base para un verdadero razonamiento científico-filosófico.

En esta perspectiva, no se trataba de cualquier tipo de observación o experimentación sino de aquello que implicaba la utilización de instrumentos técnicamente efectivos, pero no solo para potenciar la capacidad máxima de los sentidos, sino para asegurar la obtención de los datos con la mayor objetividad y precisión posible, buscando sacar a la luz aquello que aún estaba oscurecido para los sentidos. Por ello, lo más resaltante de este tipo de experimentación es que, con sus resultados, se logró superar el dualismo aristotélico dominante que servía como base para el sistema cosmológico tolemaico.

La habilidad de Galileo, como buen experimentador, no se debe solo a que reconstruyera y adaptara instrumentos tecnológicamente eficaces (como el telescopio), con el que tuvo la idea única de observar el movimiento de los cuerpos celestes, sino por su afán de encontrar precisión en la observación, especialmente, tal como lo manifiesta en el Sidereus Nuncius, en donde confiesa su adhesión a las ideas copernicanas.

Es decir, en su afán por favorecer la teoría heliocéntrica, Galileo muestra su preocupación por un conjunto de procedimientos que no estaban siendo considerados por la academia oficial, pero que le permitieron justificar su racionalidad filosófica experimental. Sin embargo, en el pensamiento galileano no bastaba la observación objetiva y precisa, sino que era muy importante correlacionar los principios matemáticos que permitían comprender y explicar los datos observados en relación, por ejemplo, al movimiento y la velocidad de los cuerpos celestes, ya que las matemáticas fueron consideradas como el lenguaje de la naturaleza. En efecto, en El Ensayador, Galileo sostiene que:

"La filosofía está escrita en ese gran libro que es el universo, el cual permanece continuamente abierto ante nuestros ojos. Pero ese libro no nos es inteligible a menos que antes aprendamos a comprender el idioma e interpretar los signos de que está compuesto. Está escrito en el idioma de las matemáticas y sus signos son triángulos, círculos y otras figuras geométricas, sin las cuales es humanamente imposible entender una sola palabra de él".

Asimismo, Galileo muestra su habilidad no solo en el conocimiento de la geometría y las 
matemáticas sino también en la aplicación de las mismas a la realidad física. Veamos lo que él mismo nos dice en su debate con los aristotélicos:

\section{“... no existiendo ninguna duda de que cuanto yo presentaba, estaba confirmado y demostrado con pruebas geométricas, al contradecirme, no advertían; tanta es la ceguera de la pasión, que contradecir la geometría es lo mismo que negar abiertamente la verdad. [Asimismo] No nos apartamos de nuestra costumbre que consiste en no afirmar como ciertas sino aquellas cosas que sabemos sin lugar a dudas que lo son, como así nos enseña nuestra filosofía y nuestras matemáticas".}

De este modo, la filosofía experimental de Galileo, tal como se puede apreciar en El Ensayador, no recurre al argumento de la tradición o de la autoridad. Más aún contrapone la autoridad con la experiencia:

“... si, para mostrar la falsedad del dicho de Aristóteles, es necesario que esos muchos no lo hayan creído ni escrito, ni yo, ni el señor Mario, ni todo el mundo junto, lo demostrarán ya nunca, pues nunca se conseguirá que esos que lo han escrito y creído vuelvan para desdecirse y renegarlo; pero digo que me parece algo nuevo el que alguien pretenda anteponer el testimonio de los hombres a lo que nos muestra la experiencia... en cuanto a la autoridad, tanto vale la de uno solo como la de cien juntos para hacer que el efecto sea cierto o no".

Con todo, lo más importante es que Galileo logra conjugar la experiencia con el razonamiento matemático, es decir, sus deducciones lógicas están articuladas con la experimentación, llegando a conjugar dialéctica, retórica y experimentos mentales. Así lo expresa en El Ensayador:
"Quieroinferir, tratandodelaciencia que por vía de demostraciones y del razonamiento humano pueden alcanzar los hombres, que cuanto más participe ésta de la perfección, tanto menor será el número de las conclusiones que prometerá enseñar y menor aún el número de las que demostrará, y en consecuencia pocos serán los que se sientan atraídos y menor aún el número de seguidores".

Aquí, no se trata de un razonamiento de matemática pura sino de un razonamiento de la física que se limita a describir las cualidades objetivas de los cuerpos, cuantitativas y mensurables, sin considerar las cualidades subjetivas del observador y mucho menos las esencias. Sin embargo, Koyré (1994) sostiene que Galileo era más platónico de lo que se podría pensar, pues asocia matemáticas con platonismo. En efecto,

"en el diálogo y los discursos nos narran la historia del descubrimiento, o, mejor dicho, del redescubrimiento del lenguaje de la Naturaleza. Nos explican la manera de interrogarla, es decir, contienen la teoría de aquella investigación experimental en la que la formulación de los postulados y la deducción de sus consecuencias precede y guía la observación. Ésta para Galileo, por lo menos, es una prueba de hecho. La nueva ciencia para él es una prueba experimental del platonismo" Koyré (1994).

En suma, con la disolución del dualismo cosmológico, Galileo nos muestra un universo abierto, indefinido y finito, unificado por un conjunto de leyes que lo gobiernan, con un mismo nivel ontológico, sin distinguir cielo y tierra y en donde se da paso a la matematización de la naturaleza.

Así, se aprecia también el surgimiento de la autonomía de la ciencia, entendida como un discurso empíricamente controlable, 
cuya pretensión es saber cómo funciona este mundo ("cómo va el cielo, no cómo se va al cielo"), sin subordinarse a ningún tipo de tradición dogmática, sea religiosa o filosófica, sino tan solo a los datos de una experiencia sensible inteligente. De ahí que también puede afirmarse que Galileo no se separa del todo de la filosofía aristotélica, pues Aristóteles anteponía las experiencias sensatas a todo tipo de razonamiento, incluyendo el científico - filosófico.

Galileo nos muestra su nuevo método, matemático-experimental, y su concepción determinista y mecanicista del mundo, el cual ya no está ordenado en función del hombre, ni mucho menos este es el fin de aquel, sino que está ordenado geométricamente, con un orden que se debe explicar con los procedimientos metodológicos de la observación y la experiencia antes establecidos. Ya no se trata de aproximarse al mundo con actitud pasiva sino con la precisión y exactitud de las matemáticas y de la geometría euclidiana para el impulso científico, tarea que luego será completada por Renée Descartes.

\section{II}

A diferencia de Galileo, Francis Bacon entiende que necesitamos comprender $y$ explicar la naturaleza, pero para dominarla y aprovecharla en función de los intereses de toda la humanidad, pero esta empresa no se puede lograr con el método y la lógica dominante. Por ello, en sus obras como el Avance del Saber (1605) y el Novum Organum (1620), Bacon rechaza el Organum aristotélico porque su método deductivo solo demuestra la validez de conocimientos ya adquiridos y además produce generalizaciones precipitadas, por lo que no puede patrocinar el progreso de la ciencia. De ahí la necesidad de una nueva lógica para crear y aumentar el conocimiento o un nuevo arte de la invención:

"Todo lo que se ha descubierto hasta ahora en las ciencias depende casi enteramente de las nociones vulgares. Para penetrar en ámbitos más recónditos de la naturaleza es necesario que tanto las nociones como los axiomas se abstraigan de las cosas por una vía cierta y segura, así como se haga un uso determinado del entendimiento mucho mejor y más seguro".

Asimismo, señala que:

"el estado actual de las ciencias no es ni afortunado ni ha experimentado un progreso; que se ha de abrir al entendimiento humano una vía completamente distinta de la conocida en el pasado y se han de procurar otras ayudas para que la mente pueda ejercer sobre la naturaleza el derecho que le corresponde" (Novum Organum, Prefacio).

Para promover este cometido, Bacon se propone, ante todo, liberarnos de aquellos "ídolos" o errores que han oscurecido la mente humana al momento de comprender y explicar la naturaleza, atentando, de este modo, en contra de una interpretación correcta de la naturaleza. Se trata de cuatro clases de "ídolos" o errores comunes que impiden el verdadero conocimiento: a) "los ídolos de la tribu", que se refiere cuando se atiende solo a las evidencias que confirman nuestras creencias y no a la evidencia que las refute. Este es un error de la naturaleza humana porque el entendimiento tiende a postular más regularidades en la naturaleza de las que realmente encuentra, a generalizar precipitadamente y a sobrestimar el valor de los casos confirmatorios; b) "los ídolos de la caverna", que son actitudes hacia la experiencia que surgen de la educación de los hombres como individuos; c) "los ídolos de la plaza o del mercado", que son distorsiones que se dan cuando el significado de las palabras quedan reducidos al sentido común impidiendo la formación de conceptos científicos; y d) "los ídolos del teatro" que son los conocimientos dogmáticos de las tradiciones filosóficas. Para nuestro autor, la tradición filosófica aristotélica pertenecía a este grupo de los ídolos del teatro.

Después de atacar a estos diversos "ídolos", Bacon muestra asumir el esquema básico del 
método aristotélico, el inductivo-deductivo, pero pone el énfasis en la etapa inductiva del procedimiento científico y considera los argumentos deductivos como clave, pero solo para confirmar las generalizaciones inductivas, pues se trata de encontrar nuevos conocimientos e invenciones.

Lord Bacon considera que la ciencia persigue el conocimiento teórico, pero no por sí mismo sino solo como medio para obtener tecnología práctica. Y para favorecer todo esto, nos propone realizar inducciones mucho más prudentes extrayendo generalizaciones seguras de la experimentación para lo cual remite a Galileo. En efecto, defiende la importancia de:

"la experimentación [que] consiste en interrogar metódicamente a la naturaleza; esta interrogación presupone e implica un lenguaje en el que formular las preguntas, así como un diccionario que nos permita leer $e$ interpretar las respuestas... para Galileo, como sabemos es... en lenguaje geométrico como debemos hablar a la naturaleza y recibir sus respuestas" (p. 153).

En este sentido, lanza sus críticas a la tradición filosófica por su falta de atención a la experiencia concreta y las aplicaciones prácticas; pero también a la tradición artesanal, aunque no por ser tecnológica, pues tiene orientación práctica y experiencial, sino por su falta de sistematización. De ahí también su rechazo a lo que denominaba "filosofía sofística" o "filosofía supersticiosa" porque parte de premisas no demostradas y porque mezcla cuestiones teológicas y metafísicas con el estudio de la naturaleza material. Más aún, considera que una parte de la filosofía racionalista tradicional (como la de Platón y los Pitagóricos) se correspondería con la filosofía supersticiosa, mientras que otra parte, concretamente el modelo aristotélico, se correspondería con la filosofía sofística que es la que pretende obtener conocimientos por medio de la deducción silogística a partir de principios no demostrados.
El método de razonamiento científicofilosófico que defiende Bacon es el inductivo, o llamado también "de diferencia", en el que se comparan situaciones en los que se da el fenómeno y situaciones en las que no se da dicho fenómeno. La causa del fenómeno tiene que ser una condición que está presente en los casos en el que el fenómeno se da, pero no en los casos en que no se da. Para explicar este método no basta, por tanto, con comparar los casos en que se da el fenómeno y buscar una condición presente en todos ellos (lo que sería el método de concordancia) sino que es preciso, además, comparar con casos negativos en los que no se da el fenómeno.

Por otro lado, propone el método de variación concomitante que compara casos en que se da el fenómeno, pero en diferentes grados, de modo que la causa del fenómeno es una condición que se da en todas las situaciones y que varía al mismo tiempo que varía la intensidad del fenómeno (directamente o inversamente), por tanto, para aplicar el método de variación concomitante no basta recopilar casos positivos, sino que hay que graduar.

Como se puede apreciar, tanto Galileo como Bacon fundan una nueva filosofía experimental al unificar el razonamiento inductivo con la experiencia y como partes constitutivas de un único método de investigación científicofilosófico, denominado también método inductivo-experimental. En efecto, Bacon lo ilustra de la siguiente manera:

"Las ciencias han sido tratadas o por los empíricos o por los dogmáticos. Los empíricos, semejantes a las hormigas, solo saben recoger y gastar; los racionalistas, semejantes a las arañas, forman telas que sacan de sí mismos; el procedimiento de la abeja ocupa el término medio entre los dos; la abeja recoge sus materiales en las flores de los jardines y de los campos, pero los transforma y los destila por una virtud que le es propia. Esta es la imagen del verdadero trabajo en la filosofía, que no se fía 
exclusivamente de las fuerzas de la humana inteligencia y ni siquiera hace de ella su principal apoyo; que no se contenta tampoco con depositar en la memoria, sin cambiarlos, los materiales recogidos en la historia natural $y$ en las artes mecánicas, sino que los lleva hasta la inteligencia modificados y transformados. Por esto todo debe esperarse de una alianza íntima y sagrada de esas dos facultades experimental $y$ racional..." (Novum Organum, p. 95).

No se trata de una mera contemplación de los hechos o simple recolección de datos empíricos, ni mucho menos de un razonamiento abstracto, sino más bien de una interpretación racional de dichos datos inteligentemente seleccionados, comparados y contrastados, ya que el verdadero conocimiento se adquiere gracias a la combinación de experiencia empírica y razonamiento. Sin embargo, Bacon valora el potencial científico de la deducción lógica en la medida que sus premisas tengan un soporte inductivo adecuado. En efecto, sabemos que Galileo también partía de hipótesis matemáticas para fundar su física matemática, deductiva y abstracta, en donde las leyes del movimiento, de la caída de los graves, etc., eran deducidas abstractamente, sin hacer uso de la noción de fuerza, sin recurrir a la experiencia de los cuerpos reales. Los "experimentos" a los que apelaba y ejecutaba eran "experimentos mentales" (Koyré).

En realidad, hay que diferenciar entre Aristóteles y sus seguidores que habrían aplicado mal el procedimiento al comenzar con los primeros principios de la deducción y no con las pruebas observacionales de la inducción, de modo que cayeron en un dogmatismo que separó la ciencia de su base empírica, pues hay que recordar que el mismo Aristóteles había insistido en que los primeros principios debían inducirse a partir de las pruebas observacionales: "No hay nada en el entendimiento que antes no haya pasado por los sentidos".

\section{CONCLUSIONES}

Si bien Galileo y Bacon se diferencian por ser uno italiano y el otro inglés, uno académico y el otro diplomático, uno científico y el otro divulgador de la ciencia, tienen importantes elementos en común que los asemejan más que suficiente, no tanto porque son contemporáneos o porque los dos fueron condenados a prisión, sino por su visión mecánica del mundo y el interés científico-filosófico que los opuso radicalmente a la filosofía aristotélica, interés que los llevó a desmarcarse de dicha tradición filosófica, así como de la medieval y la renacentista, y a defender la autonomía de la naciente ciencia moderna con su nuevo método y lógica orientada a explicar la verdad del cosmos y lograr el bien de la humanidad.

Efectivamente, tanto Galileo como Bacon critican no solo a la tradición filosófica, sino también a la autoridad eclesial y a los magos alquimistas, respectivamente, por no sustentarse precisamente en el método experimental sino en creencias ocultas a los sentidos. Los dos consideran al saber como un bien público y, por lo mismo, se oponen a que sea concebido como algo privado u oscuro. En realidad, el saber debe ser escrito en un lenguaje claro, preciso e intersubjetivo. De ahí que Galileo subraye constantemente la importancia del método experimental estrechamente ligado a la explicación racional para entender correctamente el funcionamiento mecánico de la naturaleza; y Bacon subraye, por su parte, la importancia de producir nuevos conocimientos obtenidos inductivamente para el progreso de la técnica al servicio de la sociedad.

Esta visión del quehacer de la ciencia, que comparten nuestros dos autores, se refuerza también por su visión comunitaria, anticipándose a lo que hoy denominamos comunidades de investigación, aunque este aspecto aparece más explícito en Bacon y, solamente, implícito en Galileo. Con todo, los dos consideran que el saber científico es el resultado de la cooperación dentro de una comunidad, que no solo busca la verdad de la realidad, validada intersubjetivamente, sino también alcanzar el dominio de la naturaleza para el bien de todos. 


\section{REFERENCIAS}

Bacon, F. (1988) [1605] El avance del saber. Madrid: Alianza Editorial.

Bacon, F. (2011) [1620] La Gran Restauración (Novum Organum). Aforismos sobre la interpretación de la naturaleza y el reino del hombre. Madrid: Tecnos.

Galilei, G. (1623) Il Saggiatore. (El Ensayador). En línea: http://conteudo.icmc.usp.br/pessoas/ andcarva/Il_Saggiatore.pdf

Galilei, G. (1632)Diálogossobrelosdosmáximossistemasdel mundo: Tolemaicoy Copernicano. En línea: http://bibliotecadigital.tamaulipas.gob.mx/archivos/descargas/31000000466.PDF

Galilei, G. (1610) Sidereus Nuntius. (El mensajero Sideral). En línea: http://homepages.wmich. edu/ mcgrew/Siderius.pdf

Koyré, A. (1994) Estudios de historia del pensamiento científico. Madrid: Siglo XXI.

Losse, J. (2000) Introducción histórica a la filosofía de la ciencia. Madrid: Alianza Editorial.

Reale, G. y Antiseri, D. (2005) Historia del pensamiento filosófico y científico. Barcelona: Herder.

Rossi, P. (1990) Francis Bacon: De la magia a la ciencia. Madrid: Alianza Editorial.

Fecha de recepción: 08-05-18.

Fecha de aceptación: 30-05-18. 